\title{
Política de promoção da saúde e planejamento urbano: articulações para o desenvolvimento da cidade saudável
}

\author{
Health promotion policy and urban planning: \\ joint efforts for the development of healthy cities
}

Ana Maria Girotti Sperandio ${ }^{1}$

Lauro Luiz Francisco Filho ${ }^{1}$

Thiago Pedrosa Mattos ${ }^{1}$

${ }^{1}$ Faculdade de Engenharia Civil, Arquitetura e Urbanismo, Universidade Estadual de Campinas. Av. Albert Einsten 951, Cidade Universitária. 13083-852 Campinas SP Brasil. amgspera@uol.com.br

\begin{abstract}
The National Health Promotion Policy (PNPS) defines strategies for devising inter-sectoral public policies that ensure the development of healthy cities. Urban planning constitutes a tool to improve the quality of life and enhance health promotion. Using the studies and cooperation actions conducted by the Urban Research Laboratory (LABINUR/FEC-Unicamp) as a reference, this article describes relevant aspects of the PNPS that have an interface with urban planning policies in Brazil. An increase in interdisciplinary and inter-sectoral measures related to the new PNPS after the passing of Ordinance 2.446/14 was identified, which include: mobility and accessibility; safe development (sanitation, housing and transport); healthy eating with social inclusion and reduction of poverty (community vegetable gardens); corporal activities and physical exercise and the enhancement of urban spaces. The conclusion drawn is that social participation, inter-sectoral activities and the role of the university are important aspects for the promotion of healthy cities.
\end{abstract}

Key words Health promotion, Urban planning, Healthy city
Resumo A Politica Nacional de Promoção da Saúde (PNPS) define as estratégias para a construção de políticas públicas, intersetoriais, que contribuem para o desenvolvimento de cidades saudáveis. O planejamento urbano é uma ferramenta para a melhoria da qualidade de vida, colaborando na promoção da saúde. Tomando por referência estudos e ações de cooperação desenvolvidas pelo Laboratório de Investigações Urbanas (LABINUR/FEC-Unicamp), este artigo descreve aspectos relevantes da PNPS que têm interface com as politicas de Planejamento Urbano no Brasil. Identificou-se um crescimento de dispositivos interdisciplinares e intersetoriais com a nova PNPS, através da Portaria 2.446/14, tais como: mobilidade e acessibilidade; desenvolvimento seguro (saneamento, habitação, transporte); alimentação saudável com inclusão social e diminuição da pobreza (hortas urbanas); práticas corporais e atividades físicas com a melhoria dos espaços urbanos. Conclui-se que a participação social, a intersetorialidade e a contribuição da universidade são aspectos diferenciais para promoção das cidades saudáveis.

Palavras-chave Promoção da saúde, Planejamento urbano, Cidade saudável 


\section{Introdução}

Este artigo tem como objetivo apontar as interfaces entre a promoção da saúde e o planejamento urbano quando são completados 10 anos da Política Nacional de Promoção da Saúde (PNPS) no Brasil, a de $2006^{1}$ e sua revisão em 2014, a PNaPS², reafirmando a importância da intersetorialidade e da interdisciplinaridade para o desenvolvimento das cidades saudáveis. Busca demonstrar como a inclusão de estratégias do Planejamento Urbano na Política Nacional de Promoção da Saúde ajuda no desenvolvimento da cidade saudável.

A promoção da saúde é considerada como um conjunto de ações que envolvem estratégias para a ampliação de autonomias individuais e coletivas para a vivência no território ${ }^{2}$, na direção de um estado do sentir-se bem e feliz. As ações, na perspectiva da promoção da saúde, devem respeitar princípios e valores como a participação, a autonomia e a intersetorialidade. Promover saúde é viabilizar maneiras para que os níveis de bem -estar da população se ampliem coletivamente, sendo inclusivas e acessíveis à realidade social ${ }^{1}$.

Para o desenvolvimento das estratégias de promoção da saúde é necessário se apropriar de formas multidisciplinares e interdisciplinares de modo a melhor acolher os desejos da população. É importante firmar parcerias entre gestores públicos e cidadãos para a formulação de políticas públicas na construção de uma sociedade inclusiva e equitativa que estimule: o convívio coletivo; a mobilidade e a acessibilidade sustentáveis; e o pertencimento. Estes são considerados fatores fundamentais para a equidade, a justiça social e para contribuir na criação e manutenção de cidades saudáveis ${ }^{1}$. A cidade do século XXI precisa ser promovida com base participativa, inclusiva, ouvinte e isonômica.

A cidade é dinâmica, se constituindo de um campo para onde todas as ações humanas convergem, estabelecendo uma rede em que as lutas e as expectativas de cada indivíduo ou grupo se sobrepõem de formas, às vezes, difíceis de serem separadas. Assim, seu planejamento deve ser no sentido de estruturá-la para promover a igualdade na ocupação dos espaços; potencializar a prosperidade; minimizar os riscos; estabelecer redes de comunicação e garantir a participação social e coletiva em defesa do bem comum e desenvolvimento da governança local. O Planejamento urbano deve ter como meta a qualidade de vida ${ }^{3}$.

A PNPS de $2006^{1}$ e a sua revisão, PNaPS, de 2014, pela Portaria Federal 2.446/2014² e outros documentos oficiais e referencias bibliográficos na área do urbano são estudados desde 2012 pelo Laboratório de Investigações Urbanas (LABINUR) da Faculdade de Engenharia Civil, Arquitetura e Urbanismo da Universidade Estadual de Campinas (FEC-Unicamp), por meio do Grupo de Pesquisa em Metodologias de Planejamento Urbano para Cidades Saudáveis e do Grupo de Estudos em Planejamento Urbano e Cidades Saudáveis (GEPUCS). Estes grupos têm como objetivos identificar as convergências entre o planejamento urbano e a promoção da saúde de modo a poder propor estratégias multidisciplinares para colaborar com os processos de organização e estruturação da cidade saudável, ressaltando as políticas públicas saudáveis, incluindo desejos e necessidades individuais e coletivas ${ }^{4}$, considerando princípios e valores como a participação social, a cooperação, a intersetorialidade e a governança ${ }^{5}$. Tal perspectiva aponta para o debate da Política Nacional de Promoção da Saúde nestes 10 anos, destacando aspectos que auxiliam na articulação para o desenvolvimento do planejamento urbano com enfoque na cidade saudável ${ }^{3}$.

\section{Um olhar histórico interdisciplinar entre Saúde Pública, Promoção da Saúde e Planejamento Urbano}

A interação entre as áreas da Arquitetura e da Medicina são observadas há tempos. O designer da pirâmide de Djoser - Imhotep (XXVII a.C.), no antigo Egito - era uma das pessoas responsáveis pela prática de medicina ${ }^{6}$. Na época da renascença, Leonardo Da Vinci investigou o mundo através de múltiplas áreas, como engenharia, medicina, arquitetura e outras. No auge da revolução industrial, as cidades inglesas sofriam com a falta de saneamento para a população que adensava os espaços urbanos tornando o meio propicio a epidemias.

O urbanismo nasce no início do século XX com o objetivo de resolver problemas urbanos graves, combatendo as epidemias por meio do saneamento, da drenagem de terrenos paludosos e viabilizando a mobilidade humana, melhorando a cidade através do ajardinamento, do embelezamento e da arrumação dos espaços urbanos que surgidos com o crescimento desordenado das cidades, causado pela forte expansão industrial.

O século XIX foi um período problemático por conta das ocorrências de epidemias e pela falta de infraestrutura das cidades, causadas pelo rápido crescimento das populações urbanas. Londres, por exemplo, registrou casos de cólera por volta de 1850. A esse respeito, o médico John 
Snow, na época, descobriu a relação entre as doenças epidêmicas e o espaço urbano, quando, ao mapear um surto de cólera, pôde estabelecer conexão entre suas origens e os fatores territoriais, reforçando a necessidade do saneamento básico como solução.

A Carta de Atenas $^{10}$, de 1933, surgiu como a materialização das propostas debatidas nos Congressos internacionais de Arquitetura Moderna (CIAM), realizados no início do século XX. A tentativa, a princípio, era propor medidas para o desenvolvimento urbano. Esse fato também permite perceber uma aproximação entre as áreas da saúde e urbanas, definidas com eixos como circular, trabalhar, morar e divertir.

Contudo, a primeira metade do século XX foi repleta de exemplos que fragilizaram a condição humana ${ }^{11}$ da comunidade mundial, como a Primeira Guerra (1914-1919), a Grande Recessão (1929) e, principalmente, a Segunda Guerra (1939-1945). Ao fim dos confrontos, surgiu a necessidade de uma organização internacional que priorizasse a vida, o bem-estar das pessoas e o meio em que viviam. A Carta de Ottawa (1986), a Lei brasileira No 8.080 de 1990, são documentos que incluem o debate da ação do Planejamento Urbano $^{12}$.

No Brasil da virada do século XX, eram frequentes os vínculos entre os problemas de crescimento urbano normalmente desordenado e o aumento de epidemias; tuberculose, varíola e febre amarela são alguns exemplos ${ }^{13}$. Com acirramento de ânimos no início do regime republicano aconteceu a Revolta da Vacina em $1904^{14}$.

O Planejamento Urbano surge no Brasil ${ }^{13}$ como modo de organizar adequadamente os espaços da cidade e as ações propostas. Estudos que apontam sumariamente alguns percursos como: as Reformas de Pereira Passos ${ }^{14}$, que expulsou os mais pobres das áreas centrais do Rio de Janeiro; o Plano de Avenidas para São Paulo ${ }^{15}$, cuja intenção era permitir com que a cidade tivesse mais condições de circulação; o Zoneamento ${ }^{16}$, com a característica de dividir o território urbano de forma a elencar prioridades e investimentos de áreas distintas; influência da Sociedade para Análises Gráficas e Mecanográficas Aplicadas aos complexos Sociais ${ }^{17}$, fundada pelo Padre Lebret com a finalidade de estudar a sociedade com foco no urbanismo e nas ciências humanas; os Planos Diretores Integrados ${ }^{18}$, e outros.

A promoção da saúde, como política, surge em $2006^{1}$ no Brasil, considerada como um conjunto de ações que envolvem estratégias especiais, diferenciadas, a fim de ampliar a autonomia do indivíduo e preparar, coletivamente, cidadãos que interfiram na melhoria do território. Este objetivo, de promover condições para alcançar o bem-estar ${ }^{1,2}$ e a felicidade através de mudanças na organização do território, complementa as propostas do planejamento urbano.

Para atender os objetivos deste artigo, utilizou-se a metodologia da análise de conteúdo, considerando as três fases: pré-análise; exploração; interpretação ${ }^{19}$. Para tal, foram realizadas leituras "flutuantes" que determinaram a exploração dos textos direcionados às analises das políticas: PNPS de $2006^{1}$ e a sua revisão, em $2014^{2}$.

Concomitantemente, considerou-se bibliografias que subsidiaram conceitualmente a proposta, alicerçando-se na produção científica oriunda dos projetos no LABINUR, em especial do GEPUCS.

\section{A inclusão na Política Nacional de Promoção da Saúde de estratégias do Planejamento Urbano para o desenvolvimento da cidade saudável - o período de 2006-2016}

A partir dos estudos pode-se realçar que nos dez anos (2006-2016) da PNPS se destacaram o envolvimento interdisciplinar e a interação entre áreas distintas, a Promoção da Saúde e o Planejamento Urbano.

O Estatuto da Cidade ${ }^{20}$ (Lei 10.257/01) é um instrumento da política urbana por definir que a propriedade privada deve cumprir sua função social, iniciando assim um diálogo para a construção da cidade saudável. É um ponto inicial, mas limitado, considerando-se a obrigatoriedade da realização do Plano Diretor - um mecanismo fundamental à acessibilidade acerca da participação isonômica na formulação de políticas públicas de uso e ocupação do solo ${ }^{20,21}$.

Nessa perspectiva, a PNPS de 2006 e sua reformulação de 2014 é um marco histórico da República brasileira, por participar institucionalmente na pauta do planejamento urbano disponibilizando outras ferramentas. O próprio objetivo desta Política estabelece a promoção da qualidade de vida ${ }^{1}$ como princípio, com redução da vulnerabilidade humana relacionada aos “(...) modos de viver, condições de trabalho, habitação, ambiente, educação, lazer, cultura, acesso a bens e serviços essenciais" ${ }^{2}$. Promover a qualidade da habitação e ambiente é função do urbanista, que conta com o auxílio de, ao menos, três disposições de objetivações específicas da PNPS 2006 artigos VI, VII e VIII. 
Ao estudar e refletir a reformulação realizada na PNPS de 2006 e 2014 foi possível identificar um crescimento significativo entre as políticas de promoção da saúde e da urbana, através do Estatuto da Cidade, apresentado no Quadro 1.

O Quadro 2, especifica dispositivos da reformulação da PNPS de 2014.
Enquanto estratégias, os temas prioritários da PNaPS de 2014, estreitos ao desenho e Planejamento Urbano, estão delimitadas pelas disposições normativas do Art. 10, incisos II, III, VI e VIII.

Tanto a política urbana, quanto a de promoção da saúde devem garantir a participação

Quadro 1. Aproximações entre o Estatuto da Cidade e a PNPS de 2014, LABINUR, 2016.

\begin{tabular}{|l|l|l|}
\hline \multicolumn{1}{|c|}{$\begin{array}{c}\text { Lei 10.257/2001 } \\
\text { (Estatuto da Cidade) }\end{array}$} & $\begin{array}{c}\text { Portaria 2.446/2014 } \\
\text { (PNPS 2014) }\end{array}$ & \multicolumn{1}{|c|}{ Os Dispositivos tratam } \\
\hline Art. 3o, inciso IV; Art. 41, §3 & Art. 7o, inciso III & $\begin{array}{l}\text { Mobilidade; Acessibilidade; Desenvolvimento seguro } \\
\text { (saneamento, habitação, transporte). }\end{array}$ \\
\hline Art. 1 & Art. 7o, inciso V & Bem-estar; Ambiente Saudável \\
\hline Art. 20, inciso II; Art. 45 & Art. 7o, inciso VII & Participação popular; Autonomia \\
\hline
\end{tabular}

Fonte: Brasil ${ }^{2,20}$.

Quadro 2. Características e abordagens da PNPS de 2014 como instrumentos para uma cidade saudável, 2016.

\begin{tabular}{|c|c|c|}
\hline PNaPS Portaria 2.446/14 & Característica & Abordagem \\
\hline Art. $5^{\circ}$, inciso II & Equidade & $\begin{array}{l}\text { Planejamento de ações de promoção da saúde, } \\
\text { favorecendo a construção de espaços de produção } \\
\text { social saudável, em vista aos direitos humanos. }\end{array}$ \\
\hline $\begin{array}{l}\text { Art.7o, incisos II, IV, V, VI, } \\
\text { VIII }\end{array}$ & Objetivo da PNPS/14 & $\begin{array}{l}\text { Mobilidade e acessibilidade saudável e segura; } \\
\text { promover a cultura da paz em territórios e } \\
\text { municípios; apoiar a produção de ambientes } \\
\text { saudáveis em busca do bem viver; valorizar os } \\
\text { saberes populares; autonomia para sujeitos e } \\
\text { coletividade. }\end{array}$ \\
\hline Art. $8^{\circ}$, inciso IV & Transversalidade & Ambientes Saudáveis \\
\hline Art. $9^{\circ}$, incisos II e VIII & $\begin{array}{l}\text { Intra e } \\
\text { Intersetorialidade }\end{array}$ & $\begin{array}{l}\text { Estratégias para promover a saúde; participação e } \\
\text { disseminação dos saberes }\end{array}$ \\
\hline Art. 10, incisos II, III e VIII & Prioritária & $\begin{array}{l}\text { Alimentação saudável com inclusão social e } \\
\text { diminuição da pobreza (hortas urbanas); práticas } \\
\text { corporais e atividades físicas, ciclovias); promoção } \\
\text { do desenvolvimento sustentável na cidade. }\end{array}$ \\
\hline Art. 11, inciso $\mathrm{X}$ & Pacto Interfederativo & $\begin{array}{l}\text { Intercâmbio de experiências, pesquisas e estudos que } \\
\text { visam o aperfeiçoamento tecnológico e disseminação } \\
\text { do conhecimento. }\end{array}$ \\
\hline
\end{tabular}

Fonte: Brasil2. 
social, a intersetorialidade, a autonomia, o respeito às diversidades na cidade, a solidariedade, a inclusão e a justiça social. A responsabilidade na implementação desses dispositivos não são exclusivos da abrangência do setor saúde, sendo também de outros setores e da agregação destes, enfatizando a transversalidade e a intersetorialidade. A esse respeito, a Universidade tem um papel diferencial no auxílio do desenvolvimento social, com respectivas práticas de estudo, pesquisa e monitoramento.

Esses elementos transversais à PNaPS 2014, assim como ao Estatuto da Cidade, são fundamentais para a construção de uma Cidade Saudável. Conforme Duhl e Hancock ${ }^{22}$ : Cidade saudável não é somente uma cidade com alto nível de saúde, medido pelos indicadores de mortalidade e morbidade.

Para Israel Brandão ${ }^{23}$ :

[...] Um município, para ser saudável, precisa ser amado pelas pessoas que lá habitam. Elas precisam compreender que aquele espaço lhes pertence e que, portanto, necessita ser cuidado por elas e pelos que elegem como sendo seus representantes.

No Gráfico 1 é possível perceber que a partir de 2014, para atingir uma cidade saudável, a gestão pública possui ao menos 1 (um) marco regulatório, o Estatuto da Cidade, e mais 13 (treze) dispositivos intersetoriais na PNaPS de 2014, derivadas de 3 (três) perspectivas da PNPS 2006 (VI, VII e VIII), que subsidiaram a ampliação em 2014. Conforme os dados utilizados, foi possível perceber um crescimento de 76,92\% nos dispositivos intersetoriais, entre Promoção da Saúde e Planejamento Urbano.

Elaborado a partir das experiências acadêmicas que reafirmam a interdisciplinaridade que permite a construção conceitual que instrumentalize profissionais, alicerçados no desenvolvimento científico, a Universidade tem a missão de propor investigações que atentem para propostas inovadoras nas interfaces entre o planejamento urbano e demais áreas e campos.

O resultado nessa perspectiva, nas abordagens acadêmicas experimentadas, foi incorporado nas disciplinas de Ambiente Urbano como Promotor da Saúde e de Planejamento urbano como promotor da Cidade Saudável realizadas pelo Programa de Pós-Graduação em Arquitetura, Tecnologia e Cidade da FEC-UNICAMP em 2014 e $2015^{24}$.

A realização de pesquisas interdisciplinares entre promoção da saúde e planejamento urbano, além de se constituir em sistema de investigação laboratorial (LABINUR), envolveu-se na grade curricular oferecida para formação de

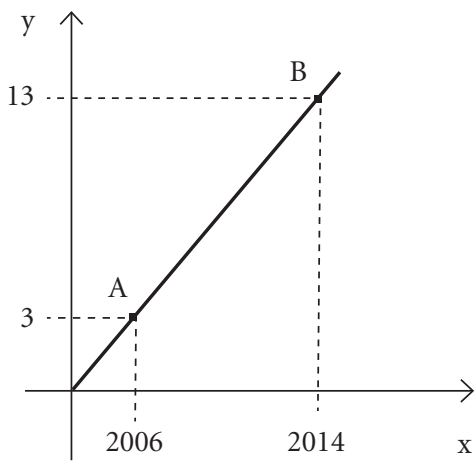

Legenda:

A - PNPS 2006 (VI, VII e VIII);

B - PNPS 2014 (Art. 5º, inciso II, Art. 7º, incisos II, IV, V, VI, VIII; Art. $8^{\circ}$, inciso IV; Art. 9º, incisos II e VII; Art. 10, incisos II, II e VIII; Art. 11, inciso X);

$\mathrm{y}$ - Dispositivos da PNPS em interfaces ao planejamento urbano;

$\mathrm{x}$ - tempo em anos

Gráfico 1. Desenvolvimento da Política Nacional de Promoção da Saúde em relação ao planejamento urbano, 2006-2014.

Fonte: Brasil ${ }^{2,20}$

profissionais. Reforçando laços do papel social da Universidade com a extensão universitária, mediante o GEPUCS. As pesquisas podem ser monitorada como o projeto de Horta Comunitária em Conchal-SP ${ }^{4}$ realizado pela FEC-UNICAMP ${ }^{4}$.

A Figura 1 demonstra como o conhecimento de planejamento urbano para cidade saudável tem sido abordado e construído na FEC-UNICAMP.

O registro desse processo pedagógico, interdisciplinar é fundamental para o desenvolvimento científico, uma vez que alicerça o conhecimento em fundações participativas, críticas, produtivas e horizontais.

A parceria em estudos que se dedicam a monitorar, teorizar, avaliar a promoção da saúde permite relações de informações e podem alcançar o desenvolvimento de projetos e práticas sociais que promovam saúde e qualidade de vida. A Universidade, em relação ao tema, precisa reforçar e ampliar cada vez mais o olhar para a interdisciplinaridade e construir novas metodologias para compartilhar saberes e interesses comuns. 

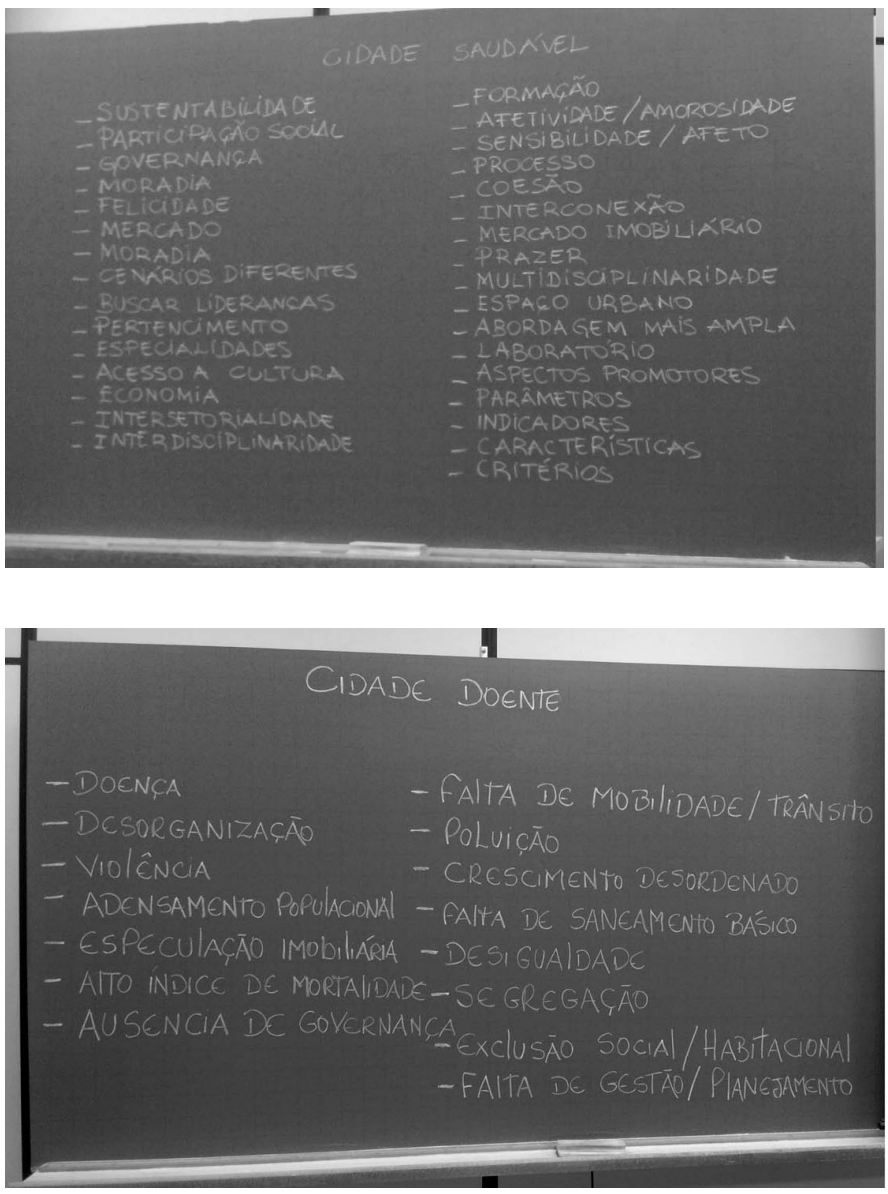

Figura 1. Conceituando-se a cidade saudável e a cidade doente na disciplina Planejamento Urbano como promotor da cidade saudável, 2015.

Fonte: Os autores.

\section{Colaboradores}

AMG Sperandio, LL Francisco Filho e TP Mattos participaram igualmente de todas as etapas de elaboração do artigo. 


\section{Referências}

1. Brasil. Ministério da Saúde (MS). Secretaria de Vigilância em Saúde. Secretaria de Atenção à Saúde. Política Nacional de Promoção da Saúde. 3a ed. Brasília: MS; 2010. p. 17-19.

2. Brasil. Ministério da Saúde (MS). Secretaria de Vigilância à Saúde. Gabinete do Ministro. Portaria No 2.446, de 11 de Novembro de 2014. Redefine a Política Nacional de Promoção da Saúde (PNPS). Diário Oficial da União 2014; 12 nov.

3. Sperandio AMG. Editorial. Revista Intellectus 2012; $\operatorname{VIII}(22): 2-4$.

4. Sperandio AMG, Mattos TP, Francisco Filho LL, Fávero E, Dias A, Ribeiro MM, Manfrinato T. A horta comunitária como ferramenta de ocupação de vazio urbano e promotora de práticas saudáveis. PARC 2015; 6(3):205215.

5. Westphal MF. O movimento cidades/municípios saudáveis: um compromisso com a qualidade de vida. Cien Saude Colet 2000; 5(1):35-51.

6. Mead PC. Architecture as Environmental Medicine. In: Proceedings of the ACSA Annual Meeting. Association of Collegiate Schools of Architecture; 1996. p. 188.

7. Jornal da Unicamp, 29/07/2014 a 04/08/2014, p.4. [acessado 2016 mar 3]. Disponível em: http://LABINUR.unicamp.br/unicamp/sites/default/files/jornal/ paginas/ju_568_pagina_04_0.pdf

8. Choay F. O urbanismo: utopias e realidades, uma antolo-

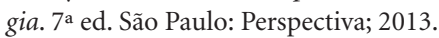

9. Freitas CM. Problemas ambientais, saúde coletiva e ciências sociais. Ciência e Saúde Coletiva 2003; 8(1):137-150.

10. IPHAN (Instituto do Patrimônio Histórico e Artístico Nacional). Carta de Atenas. Assembleia do Congresso Internacional de Arquitetura Moderna. Novembro 1933. [acessado $2016 \mathrm{fev}$ 5]. Disponível em: portal. iphan.gov.br/upload/ckfinder/arquivos/carta $\% 20 \mathrm{de} \%$ 20Atenas\%201933.pdf

11. Arendt H. A condição humana. Rio de Janeiro: Forense; 1981.

12. Sperandio AMG Mattos TP, Fávero E, Dias ATC. Planejamento urbano e saúde pública: (re)visitando uma história contextualizada. Revista Intellectus No 28, 2014; 28 (Volume especial de planejamento urbano saudável):18. [acessado 2015 nov 4]. Disponível em: http:// LABINUR.revistaintellectus.com.br/DownloadArtigo. ash $x$ ? $\operatorname{codigo}=381$

13. Elias N, Scotson J. Os estabelecidos e os outsiders: sociologia das relações de poder a partir de uma pequena comunidade. Rio de Janeiro: Zahar; 2000.

14. Leme MCS. A formação do pensamento urbanístico no Brasil, 1895-1965. In: Leme MCS, organização. Urbanismo no Brasil: 1895-1965. São Paulo: Studio Nobel; 1999. p. 22.

15. Leme MCS. Revisão do Plano de Avenidas: um estudo sobre o planejamento urbano em São Paulo, 1930 [tese]. São Paulo: Universidade de São Paulo; 1990.
16. Borges MV. O zoneamento na cidade do Rio de Janeiro: gênese, evolução e aplicação. Rio de Janeiro: Universidade Federal do Rio de Janeiro; 2007.

17. Valladares LP. A invenção da favela: do mito de origem à favela.com. Rio de Janeiro: Editora FGV; 2005.

18. Villaça F. As ilusões do plano diretor. 2005. [acessado 2015 nov 4]. Disponível em: http://LABINUR.flaviovillaca.arq.br/pdf/ilusao_pd.pdf;

19. Caregnato RCA, Mutti R. Pesquisa qualitativa: análise de discurso versus análise de conteúdo. Texto contexto enferm 2006; 15(4):682-683.

20. Brasil. Lei n. 10.257, de 10 de julho de 2001. Regulamenta os arts. 182 e 183 da Constituição Federal, estabelece diretrizes gerais da política urbana e dá outras providências. Diário Oficial da União 2001; 11 jul.

21. Brasil. Constituição da República Federativa do Brasil de 1988. Diário Oficial da União 1988; 5 out.

22. Duhl LJ, Hancock T. A guide to assessing healthy cities. Geneva: WHO Healthy Cities Project Office; 1988.

23. Brandão IR. Na trilha do município saudável. In: Organização Pan-Americana de Saúde (OPAS). Políticas integradas em rede e a construção de espaços saudáveis: boas práticas para a iniciativa do Rostos, Vozes e Lugares. Brasília: OPAS; 2010. p. 37-38.

24. Sperandio AMG. Ementa. In: Disciplina Tópicos: Ambiente urbano como promotor da saúde. Campinas: Programa de Pós-Graduação em Arquitetura, Tecnologia e Cidade, da Faculdade de Engenharia Civil, Arquitetura e Urbanismo (FEC-UNICAMP); 2014.
Artigo apresentado em 05/02/2016 Aprovado em 26/04/2016 Versão final apresentada em 28/04/2016 
